\title{
MR Visualization of Retrograde Aortic Flow Pattern in a Patient with Recurrent Embolic Strokes
}

\author{
Hashim Javarov ${ }^{a}$ Thomas Henzler ${ }^{\mathrm{a}}$ Henrik Michaely $^{\mathrm{a}}$ \\ Michael G. Hennerici ${ }^{b}$ Anastasios Chatzikonstantinou ${ }^{b}$ \\ Stefan O. Schoenberg ${ }^{a}$ Christian Fink $^{a}$ \\ anstitute of Clinical Radiology and Nuclear Medicine, and bepartment of Neurology, \\ University Medical Center Mannheim, Medical Faculty Mannheim, Heidelberg University, \\ Mannheim, Germany
}

A 76-year-old patient with a history of recurrent left-hemispheric strokes within the last two years was referred for ECG-gated CT angiography (CTA) of the chest for the assessment of aortic plaque burden as a potential source of embolic stroke episodes. CTA revealed significant atherosclerotic plaques in the descending aorta as well as a moderate aortic isthmus stenosis with kinking $(\mathbf{A}-\mathbf{C})$. Significant carotid artery stenosis was excluded by contrastenhanced MRA. Additional time-resolved three-dimensional phase-contrast MRI showed a retrograde flow pattern towards the supra-aortic vessels (D-F) suggesting recurrent embolism from the plaques in the descending aorta (see also supplementary online video, www. karger.com/doi/10.1159/000338540).

This case illustrates that visualization of flow patterns with time-resolved three-dimensional phase-contrast MRI provides additional information in patients with embolic strokes in whom ECG-gated CTA revealed significant plaque burden in the aortic arch and descending aorta.

\section{Disclosure Statement}

All authors declare that they have no competing financial, funding or contributorship interests. 
Ex X T R R A

Cerebirovascular

Diseases
Cerebrovasc Dis Extra 2012;2:24-25

DOI: $10.1159 / 000338540$

Published online: April 27, 2012

2012 S. Karger AG, Basel

www.karger.com/cee

Javarov et al.: MR Visualization of Retrograde Aortic Flow Pattern
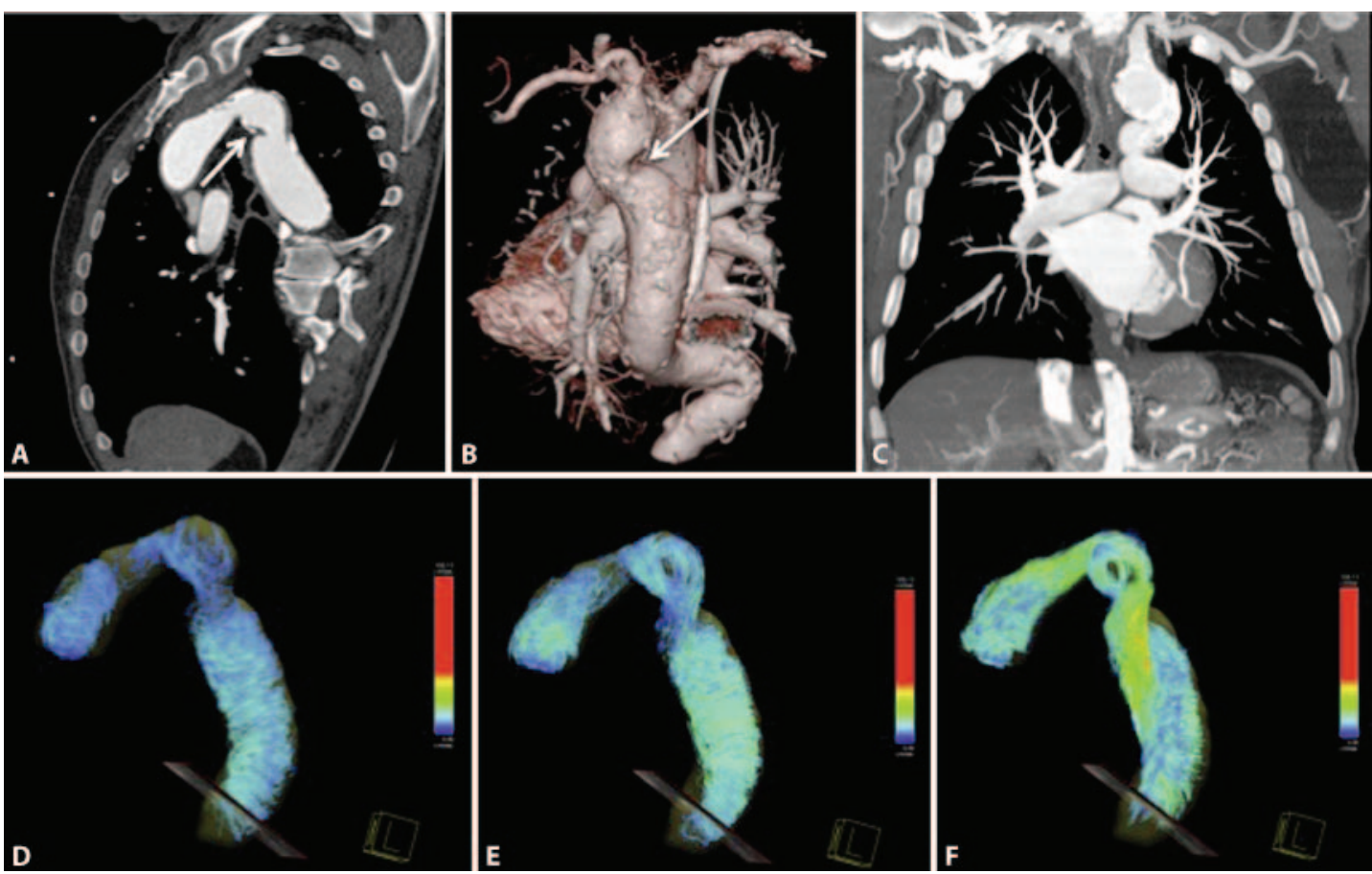